\title{
Are exercise programs an effective treatment for children with cystic fibrosis?
}
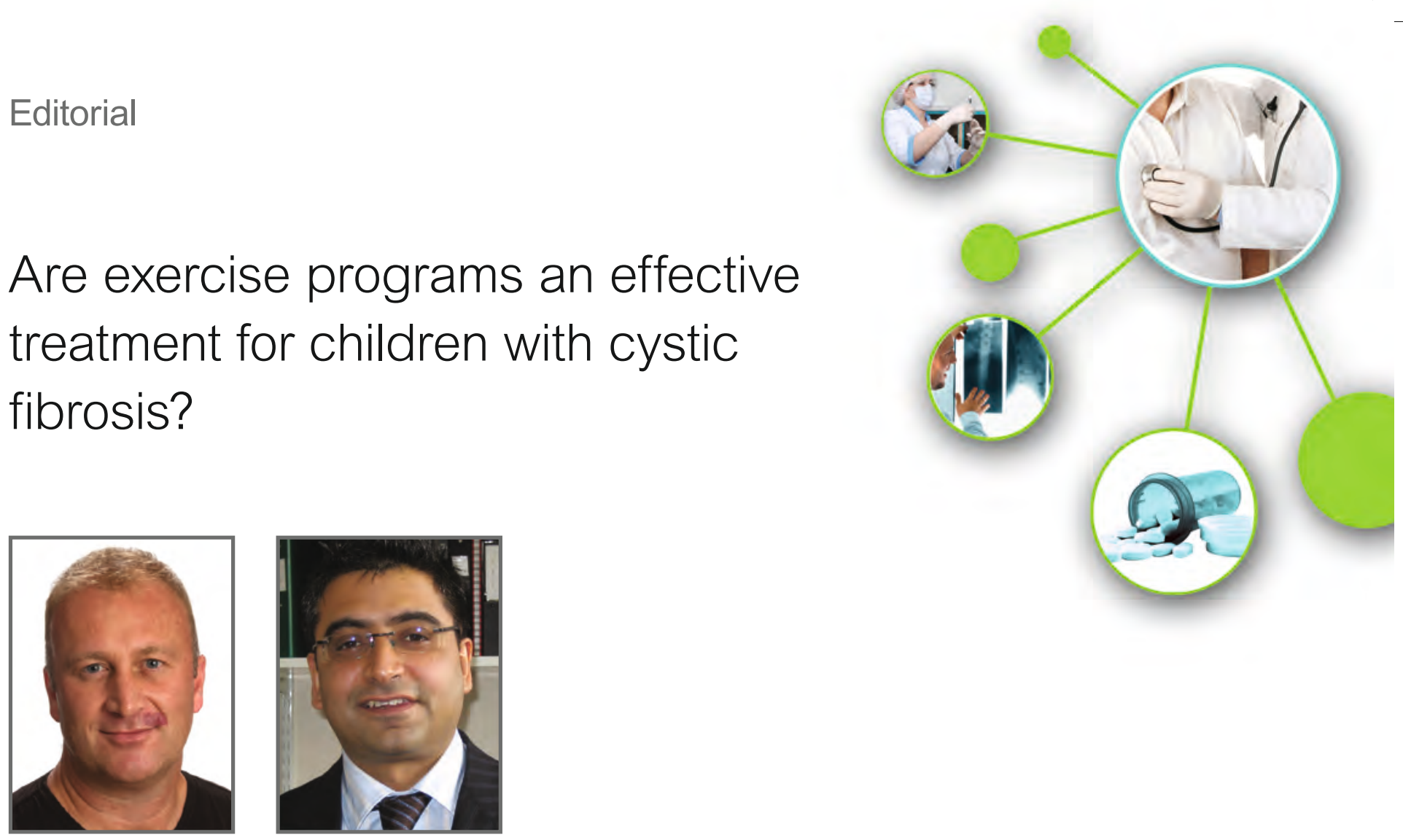

Sean J Ledger*1,2 \& Paul Aurora ${ }^{1,2}$

6

"...recent pilot studies in subjects with more advanced disease, where the exercise intervention runs over a longer period, have implied marked improvements in a variety of outcomes..."

Why could exercise be beneficial in cystic fibrosis?

Cystic fibrosis (CF) is a genetically inherited, autosomal recessive disease that is caused by a mutation in the gene encoding the cystic fibrosis transmembrane conductance regulator protein. As a result of this deficit, multiple organs, primarily the lungs, pancreas and digestive system, become congested with thick, sticky mucus resulting in infections and inflammation. Despite advances in medical care, life expectancy remains shortened, with $85 \%$ of CF-related deaths caused by lung disease [1]. Exercise capacity in this population may be limited by reduced lung function, nutritional status, metabolic deficits and peripheral muscle function.

Extensive research into the effect of exercise training and physical activity in the healthy child has demonstrated physiological and quality-oflife benefits. Guidelines for exercise testing, prescription and training for healthy children are also well documented. However, by comparison, there is much less evidence demonstrating that children with CF achieve the same level of benefits as their healthy peers, and guidelines for exercise are still being developed. Most available evidence relates to short-term benefits of exercise, with a particular dearth of good-quality longitudinal studies.

Current guidelines for the physiotherapy management of children and adults with CF suggest that regular exercise and physical activity be prescribed in conjunction with airway clearance and inhalation therapy (ACT) [2]. The rationale is that exercise may enhance sputum clearance [3], improve or maintain lung function [4], reduce breathlessness [5], increase aerobic capacity and muscle strength [6], and improve bone health [7] and quality of life [8]. Regular adherence to exercise and ACT is variable in both adults $[9,10]$ and children [11], with time required to complete ACT, nutritional status and progression of the 
“...exercise may enhance sputum clearance, improve or maintain lung function, reduce breathlessness, increase aerobic capacity and muscle strength, and improve bone health and quality of life..." disease the key factors that influence exercise capacity, and willingness to participate in any form of exercise or physical activity.

A 2008 Cochrane review by Bradley and Moran evaluated the effects of exercise training in seven randomized controlled trials that involved 231 adults and children with cystic fibrosis [12]. They identified studies that examined either the short- and long-term effects of aerobic or anaerobic exercise, or a combination of both. The authors concluded that there was an insufficient number of studies published to date to clearly define the benefits of exercise in CF. However, exercise was acknowledged to be an important component of the current management of individuals with CF and should not be discouraged.

Two nonsystematic reviews have examined the effects of supervised, unsupervised and part-supervised exercise programs in children with CF in hospital-, outpatient- and homebased settings $[13,14]$. In addition to evaluating the effects of aerobic exercise on peak oxygen consumption $\left(\mathrm{VO}_{2 \text { Peak }}\right)$, high intensity anaerobic training and muscle strength training programs were also evaluated. Exercise programs produced similarly beneficial results in males and females. Endurance training was the most popular intervention, and was found to be effective at increasing lung function and $\mathrm{VO}_{2 \text { Peak }}$, and improving breathlessness and quality of life, while strength training improved muscle strength and size, and weight gain. High intensity anaerobic training or interval training, which involved short periods of intense exercise followed by short recovery periods, showed improvements in $\mathrm{VO}_{2 \text { Peak }}$ and power output. The reviewers suggested that a comprehensive exercise-training program should include a combination of modalities. Supervised and in-patient programs for children with CF produced the most improvements in exercise parameters, with less dramatic effects noted in part-supervised or unsupervised programs.

\section{How should exercise be prescribed in CF?}

Exercise programs by design should include three core components: exercise testing, exercise prescription and exercise training. Comprehensive maximal and/or submaximal exercise tests, such as a cardiopulmonary exercise test (CPET) or functional field-based incremental walk/run tests (ISWT), should be performed to monitor for exercise-induced limitations before exercise is prescribed, and exercise training is implemented in children with $\mathrm{CF}$ [15]. However, a survey of exercise testing and training programs in UK-based CF centers reported that exercise tests, such as CPET, are limited to a few specialist centers, and even they are underused [16].

The advantage of using CPET to determine a safe, individualized exercise prescription in children with CF is that the test measures gas flow, $\mathrm{VO}_{2 \text { Peak }}, \mathrm{CO}_{2}$ production, respiratory exchange ratio, pulse oximetry and heart rate during exercise. When combined with the estimated work rate achieved during the test, variables such as anaerobic threshold and minute ventilation relative to $\mathrm{CO}_{2}$ exhalation can be calculated [17]. These outcomes are useful in determining exercise limitations such as pulmonary hypertension, exercise induced hypoxemia and cardiac arrhythmias. The results should then be used to determine target heart rates and/or ventilatory threshold exercise training ranges for the child. In the absence of CPET, field-based test results should be performed.

Based on the results of exercise tests, frequency, intensity, time and type exercise principles should be used to guide an age-appropriate, individualized exercise training program for the child. Dependent on the aims of the training program, clinicians could prescribe aerobic, anaerobic or muscle strength training or a combination of these for the child [14]. It should be noted that guidelines for exercise prescription and training in children with CF require further development.

What is new in children with more severe disease, and where do we go from here? In the last 5 years there have been three novel, 12-month observational studies that have explored an alternative model of physiotherapy care in small groups of sicker children with moderate-tosevere CF. Children who have required $>40$ days of intravenous (iv.) antibiotic treatment in the previous year were included in the programs, and data from the intervention year were compared with the previous year's data. Each program provided regular supervised, individualized exercise training in an outpatient [18] or outreach setting $[19,20]$, in addition to current specialist CF care.

In the 2008 Brisbane study, Black et al. ( $n=10$ ) demonstrated a $48 \%$ reduction in iv. antibiotic requirement, and a significant increase in lung function and $20 \mathrm{~m}$ ISWT scores [18]. Urquhart et al. $(\mathrm{n}=12)$ demonstrated a $17 \%$ 
reduction in iv. antibiotic requirement and cost savings of approximately UK£66,384 to the hospital. In addition, children improved their ISWT scores, quality of life scores measured by the disease-specific Cystic Fibrosis Questionnaire (CFQ), and there was a trend towards improved lung function [19].

In our own CF unit at Great Ormond Street Hospital for Children (London, UK), the Frequent Flyer Programme ( $\mathrm{n}=16)$ was undertaken as a quality improvement initiative [20]. Our program showed a $25 \%$ reduction in iv. antibiotic requirement and approximately UK£113,570 cost benefit to the hospital. CPET and ISWT scores were significantly improved and the majority of children on the program maintained their lung function and growth outcomes. The children's CFQ scores showed only marginal improvements in quality of life, although an end of program questionnaire suggested that quality of life had been improved. Feedback from both children and parents was very encouraging, with families reporting that their children had been able to spend more time at home and school and experienced less of a dip in their general quality of health. Children reported they were now able to exercise at the same level or sometimes even higher than their peers.

These programs present an interesting new model of physiotherapy care focused on incorporating structured, supervised exercise programs into children's CF management. As observational studies, they should not be overinterpreted, particularly with regard to mechanism. It is plausible that the pilot programs offered clinicians an opportunity to monitor children more closely for subtle changes in their clinical status, and that it was this closer monitoring rather than the exercise intervention that led to improved outcomes. Nonetheless, the key findings from these three studies are that most subjects had a quantifiable improvement in health outcomes while on the exercise program and that a concurrent reduction in other therapies generated

\section{References}

1 Mehta G, Macek M Jr, Mehta A. Cystic fibrosis across Europe: EuroCareCF analysis of demographic data from 35 countries (on behalf of the European Registry Working Group). J. Cyst. Fibros. 9(Suppl. 2), S5-S21 (2010).

2 Kerem E, Conway S, Elborn S, Heijerman H, Consensus C. Standards of care for patients

savings that outweighed the cost of the exercise intervention.

The above three programs all focused on individuals with moderate-to-severe lung disease. The question then arises regarding the impact a structured program could have on subjects with milder disease, and here the evidence is currently lacking. A number of randomized trials are currently collecting data, including one European collaborative study and another study at our own center. The aim of these studies is to quantify both health improvements and the overall cost impact of extending such initiatives more widely across pediatric CF care.

In conclusion, most current evidence regarding the benefit of regular exercise in children with CF has focused on the impact of exercise upon defined surrogate variables over relatively short timescales. More recent pilot studies in subjects with more advanced disease, where the exercise intervention runs over a longer period, have implied marked improvements in a variety of outcomes, and crucially a reduction in other healthcare needs that suggest exercise could be a cost-neutral or even cost-saving intervention in selected individuals. Well-structured randomized trials in a broader range of subjects are ongoing, but until then, a CF clinician/physiotherapist is required to make a clinical judgment on the value of exercise prescription in the CF clinic. The balance of evidence currently suggests that an exercise prescription is likely to be of benefit to at least some of these patients.

Financial \& competing interests disclosure

The authors have no relevant affiliations or financial involvement with any organization or entity with a financial interest in or financial conflict with the subject matter or materials discussed in the manuscript. This includes employment, consultancies, honoraria, stock ownership or options, expert testimony, grants or patents received or pending, or royalties.

No writing assistance was utilized in the production of this manuscript.

with cystic fibrosis: a European consensus. J. Cyst. Fibros. 4(1), 7-26 (2005).

3 Salh W, Bilton D, Dodd M, Webb AK. Effect of exercise and physiotherapy in aiding sputum expectoration in adults with cystic fibrosis. Thorax 44(12), 1006-1008 (1989).

4 Paranjape SM, Barnes LA, Carson KA, von Berg K, Loosen H, Mogayzel PJ Jr. Exercise
"The aim of these studies is to quantify both health improvements and the overall cost impact of extending such initiatives more widely across pediatric cystic fibrosis care." 


\section{Editorial | Ledger \& Aurora}

children with cystic fibrosis: a randomized controlled trial. Chest 126(4), 1204-1214 (2004).

7 Hind K, Truscott JG, Conway SP. Exercise during childhood and adolescence: a prophylaxis against cystic fibrosis-related low bone mineral density? Exercise for bone health in children with cystic fibrosis. J. Cyst. Fibros. 7(4), 270-276 (2008).

8 Schmidt AM, Jacobsen U, Bregnballe $\mathrm{V}$ et al. Exercise and quality of life in patients with cystic fibrosis: a 12 -week intervention study. Physiother. Theory Pract. 27(8), 548-556 (2011).

9 White D, Stiller K, Haensel N. Adherence of adult cystic fibrosis patients with airway clearance and exercise regimens. J. Cyst. Fibros. 6(3), 163-170 (2007).

10 Flores JS, Teixeira FA, Rovedder PM, Ziegler B, Dalcin Pde T. Adherence to airway clearance therapies by adult cystic fibrosis patients. Respir. Care 58(2), 279-285 (2013).
11 Prasad SA, Cerny FJ. Factors that influence adherence to exercise and their effectiveness: application to cystic fibrosis. Pediatr. Pulmonol. 34(1), 66-72 (2002).

12 Bradley J, Moran F. Physical training for cystic fibrosis. Cochrane Database Syst. Rev 1, CD002768 (2008).

13 van Doorn N. Exercise programs for children with cystic fibrosis: a systematic review of randomized controlled trials. Disabil. Rehabil. 32(1), 41-49 (2010).

14 Williams CA, Benden C, Stevens D, Radtke T. Exercise training in children and adolescents with cystic fibrosis: theory into practice. Int. J. Pediatr. 2010, doi:10.1155/2010/670640 (2010).

15 Stevens D, Williams CA. Exercise testing and training with the young cystic fibrosis patient. J. Sports Sci. Med. 6, 286-291 (2007).

16 Stevens D, Oades PJ, Armstrong N, Williams CA. A survey of exercise testing and training in UK cystic fibrosis clinics. J. Cyst. Fibros. 9(5), 302-306 (2010).
17 Radtke T, Stevens D, Benden C, Williams CA. Clinical exercise testing in children and adolescents with cystic fibrosis. Pediatr. Phys. Ther. 21(3), 275-281 (2009).

18 Black R, Tallis M, Urquhart D, Harris M, Cooper D, Dakin C. Intensive out-patient physiotherapy - an approach to optimising lung health in childhood cystic fibrosis. Pediatr. Pulmonol. 44, 391-392 (2009).

19 Urquhart D, Sell Z, Dhouieb E et al. Effects of a supervised, outpatient exercise and physiotherapy programme in children with cystic fibrosis. Pediatr. Pulmonol. 47(12), 1235-1241 (2012).

20 Ledger SJ, Owen E, Prasad SA, Goldman A, Willams J, Aurora P. A pilot outreach physiotherapy and dietetic quality improvement initiative reduces IV antibiotic requirements in children with moderatesevere cystic fibrosis. J. Cyst. Fibros. doi:10.1016/j.jcf.2013.01.003 (2013) (Epub ahead of print). 